\title{
On the multiplicity of a stochastic vector process
}

Harald Cramér

\section{Introduction}

The object of this note is to prove a theorem on the multiplicity of a stochastic vector process. Before stating the theorem, it will be necessary to give some introductory remarks.

We shall be concerned with complex-valued random variables defined on a fixed probability space. Any random variable $x$ considered will be assumed to have zero mean and a finite variance:

$$
E x=0, \quad E|x|^{2}<\infty .
$$

It is well known that all random variables satisfying these conditions can be regarded as elements in a Hilbert space, if the inner product of two elements $x$ and is defined by the relation

$$
(x, y)=E x \bar{y} \text {. }
$$

A stochastic vector process of finite dimensionality $q$ will be denoted

$$
\mathbf{x}(t)=\left\{x_{1}(t), \ldots, x_{q}(t)\right\}
$$

where the components $x_{n}(t)$ are random variables depending on a real parameter $t$, which may be regarded as representing time. By $H(\mathbf{x})$ we denote the Hilbert space spanned by the random variables $x_{n}(u)$ for all $n$ and all real $u$ :

$$
H(\mathbf{x})=\mathscr{S}\left\{x_{n}(u), n=1, \ldots, q,-\infty<u<+\infty\right\}
$$

while $H(\mathbf{x}, t)$ is the subspace of $H(\mathbf{x})$ spanned by the same $x_{n}(u)$ for all $u \leqq t$. The projection operator in $H(\mathbf{x})$ with range $H(\mathbf{x}, t)$ will be denoted by $P_{t}$.

For any element $z$ of $H(\mathbf{x})$ we write

$$
z(t)=P_{t} z
$$

$z(t)$ will then be the random variable of a stochastic process with orthogonal increments such that

$$
E z(t)=0, \quad E|z(t)|^{2}=F(t),
$$


where the variance function $F(t)$ is never decreasing, everywhere continuous to the left provided $(A)$ and $(B)$ below are satisfied, and such that $F(-\infty)=0$, $F(+\infty)=E|z|^{2}$. The Hilbert space spanned by the $z(u)$ for all real $u$ is a cyclic subspace of $H(\mathbf{x})$ which we denote by $C(z)$, while $C(z, t)$ is the subspace spanned by the $z(u)$ for all $u \leqq t$. It is known that every element of $C(z)$ will be of the form

$$
\int_{-\infty}^{\infty} c(t) d z(t)
$$

where $c(t)$ is a nonrandom function belonging to $L_{2} F(t)$, and the stochastic integral is defined as an integral in quadratic mean.

In the set of all $z$, and in the set of the corresponding variance functions $F(t)$, we introduce a partial ordering by writing $z_{1}>z_{2}$ and $F_{1}>F_{2}$ whenever $F_{2}$ is absolutely continuous with respect to $F_{1}$. Every variance function defines in the well-known way a measure on the real axis, and $F_{2}$ is absolutely continuous with respect to $F_{1}$ if and only if every set of $F_{1}$ measure zero is also $F_{2}$ measure zero. If simultaneously $F_{1} \succ F_{2}$ and $F_{2} \succ F_{1}$ we say that $F_{1}$ and $F_{2}$ are equivalent, and also the corresponding $z_{1}$ and $z_{2}$. All variance functions $F$ equivalent to a given $F_{1}$ will be said to form the equivalence class of $F_{1}$.

We shall consider a stochastic vector process $\mathbf{x}(t)$ satisfying the following two conditions (A) and (B):

(A) The limits $x_{n}(t \pm 0)$ exist for all $n$ and $t$ as limits in the norm of $H(\mathbf{x})$, and $x_{n}(t-0)=x_{n}(t)$.

(B) The limiting space $H(\mathbf{x},-\infty)$ contains only the zero element of $H(\mathbf{x})$.

It follows from (A) that the Hilbert space $H(\mathbf{x})$ is separable, and consequently the subspace $H(\mathrm{x}, t)$, which is never decreasing when $t$ increases, has at most an enumerable number of discontinuities. When (B) is satisfied, the $\mathbf{x}(t)$ process is called purely nondeterministic (or linearly regular).

When (A) and (B) are satisfied, it is known that it is possible to find a finite or infinite sequence of elements of $H(\mathbf{x})$

$$
z_{1} \succ z_{2} \succ \ldots>z_{N}
$$

such that the corresponding cyclic subspaces $C\left(z_{n}\right)$ are mutually orthogonal, and such that $H(\mathbf{x})$ is the vector sum of all these orthogonal cyclic subspaces:

$$
H(\mathbf{x})=C\left(z_{1}\right) \oplus \ldots \oplus C\left(z_{N}\right) .
$$

The number $N$ and the equivalence classes of the corresponding variance functions $F_{1}, \ldots, F_{N}$ are uniquely defined by the given $\mathbf{x}(t)$ process, and $N$ is called the multiplicity of the process. Applying the projection $P_{t}$ we obtain the relation

$$
H(\mathbf{x}, t)=C\left(z_{1}, t\right) \oplus \ldots \oplus C\left(z_{N}, t\right)
$$


for every $t$. As $x_{n}(t)$ is an element of $H(\mathbf{x}, t)$, this gives the representation

$$
x_{n}(t)=\sum_{r=1}^{N} \int_{-\infty} c_{n, r}(t, u) d z_{r}(u) .
$$

This shows how the components of the $\mathbf{x}(t)$ process can be regarded as additively built up by $N$-dimensional innovation elements $\left\{d z_{1}(u), \ldots, d z_{N}(u)\right\}$ associated with every instant $u$ in the "past and present" from the point of view of the instant $t$.

The basic facts of multiplicity theory given above are stated here in the form in which they occur in my papers [1-3].

\section{A theorem on multiplicity}

Theorem. Let $\mathbf{x}(t)$ be a given vector process satisfying (A) and (B), and suppose that the component $x_{n}(t)$, regarded as a one-dimensional process, has finite multiplicity $N_{n}$. Then the multiplicity of the $\mathbf{x}(t)$ process is $N \leqq \sum_{n=1}^{q} N_{n}$.

I have stated this theorem without proof in a paper of 1961 [1, p. 265]. Although the theorem has been referred to in the literature [e.g. 4, p. 228], no proof has been published, as far as I know. It may be of some interest to give a complete proof. As stated in my paper just quoted, the proof is slightly more involved than may possibly be expected. The proof will depend on two Lemmas.

Lemma 1. Let $z_{1}, \ldots, z_{n}$ and $w$ be elements of $H(\mathbf{x})$ such that the cyclic subspaces $C\left(z_{1}\right), \ldots, C\left(z_{n}\right)$ are mutually orthogonal. Then it is possible to find an element $z_{n+1}$ of $H(x)$ such that $C\left(z_{n+1}\right)$ is orthogonal to $C\left(z_{r}\right)$ for $r=1, \ldots, n$, and such that the vector sum

$$
C\left(z_{1}\right) \oplus \ldots \oplus C\left(z_{n+1}\right)
$$

includes the cyclic subspace $C(w)$.

Let $w_{r}$ be the projection of $w$ on $C\left(z_{r}\right)$. Then define $z_{n+1}$ by

$$
\begin{gathered}
w=w_{1}+\ldots+w_{n}+z_{n+1}, \\
P_{t} w=P_{t} w_{1}+\ldots+P_{t} w_{n}+P_{t} z_{n+1} .
\end{gathered}
$$

Here $w_{r}$ and $P_{t} w_{r}$ belong to $C\left(z_{r}\right)$, while $z_{n+1}$ is orthogonal to $C\left(z_{r}\right)$ for all $r=1, \ldots, n$. It follows $\left[1\right.$, p. 259] that $C\left(z_{n+1}\right)$ is orthogonal to all these $C\left(z_{r}\right)$.

Now every element of $C(w)$ is the limit of a convergent sequence of finite linear combinations of the $P_{t} w$. According to the above relation every such linear combination is a sum of finite linear combinations of elements of $C\left(z_{1}\right), \ldots, C\left(z_{n+1}\right)$, and since these subspaces are mutually orthogonal, the limit of any convergent sequence of this kind will be a sum of elements of the same subspaces. Thus the 
vector sum (3) includes every element of $C(w)$, and the Lemma is proved. Note that, in the case when $w$ belongs to the vector sum of $C\left(z_{1}\right), \ldots, C\left(z_{n}\right)$, the $z_{n+1}$ defined in the above proof is equal to zero.

It will be seen without difficulty that, by repeated application of this Lemma to the vector process $\mathbf{x}(t)$ satisfying the conditions of the Theorem, we can find $N=\sum_{n=1}^{q} N_{n}$ elements $z_{1}, \ldots, z_{N}$ of $H(\mathbf{x})$, some of which may be equal to zero, such that the corresponding cyclic subspaces are all mutually orthogonal, and the condition (2) will be satisfied. But the condition (1) may not be satisfied, and in order to complete the proof of the Theorem we shall require the following Lemma.

Lemma 2. If $z_{1}, \ldots, z_{N}$ are a finite number of elements of $H(\mathbf{x})$ such that the cyclic subspaces $C\left(z_{n}\right)$ are all mutually orthogonal, and the relation (2) is satisfied, we can find $N$ elements $w_{1}, \ldots, w_{N}$ of $H(\mathbf{x})$, some of which may be equal to zero, such that the cyclic subspaces $C\left(w_{n}\right)$ are all mutually orthogonal, and we have

$$
\begin{gathered}
w_{1} \succ w_{2} \succ \ldots \succ w_{N} \\
H(\mathbf{x})=C\left(w_{1}\right) \oplus \ldots \oplus C\left(w_{N}\right) .
\end{gathered}
$$

This Lemma being proved, it will be seen that the truth of the Theorem follows from the remark made before stating the Lemma.

Consider the variance functions $F_{n}$ corresponding to the $z_{n}$ given in the Lemma. The never decreasing function $\mathbf{F}(t)=\sum_{n=1}^{N} F_{n}(t)$ defines a measure on the real axis, which we shall use throughout the proof of the Lemma. Expressions like 'measurable', 'almost everywhere (a.e.)', etc., will always refer to the $\mathbf{F}$ measure.

We have $\mathbf{F} \succ F_{n}$ for every $n$, so that the Radon-Nikodym derivative $F_{n}^{\prime}=$ $d F_{n} / d \mathbf{F}$ will be defined a.e. and measurable. Note that, if $t$ is a discontinuity point of some of the $F_{n}$, we shall have $F_{n}^{\prime}(t)>0$ for every $F_{n}$ which is discontinuous at $t$, and $F_{n}^{\prime}(t)=0$ for the other $F_{n}$. For $n, r=1, \ldots, N$ we now define a function $g_{n, r}(t)$ by writing for $n \geqq r$

$$
g_{n, r}(t)= \begin{cases}1 & \text { if } F_{n}^{\prime}(t)>0 \text { and there are exactly } r-1 \text { positive } \\ & \text { among } F_{1}^{\prime}(t), \ldots, F_{n-1}^{\prime}(t), \\ 0 & \text { otherwise, }\end{cases}
$$

and $g_{n, r}(t)=0$ for all $t$ when $n<r$. Then $g_{n, r}(t)$ will be defined a.e. and measurable. It will be seen that $g_{n, r}(t)$ has the following three properties a.e.

(6) For given values of $n$ and $t$, we have $g_{n, r}(t)=1$ for at most one $r$.

(7) For given values of $r$ and $t$, we have $g_{n, r}(t)=1$ for at most one $n$.

(8) If $g_{n, r}(t)=1$ and $n \geqq r>1$, there is exactly one $m<n$ such that $g_{m, r-1}(t)=1$.

The sum of stochastic integrals

$$
w_{r}=\sum_{n=1}^{N} \int_{-\infty}^{\infty} g_{n, r}(u) d z_{n}(u)
$$


is a well-defined element of $H(\mathbf{x})$, and we have

$$
\begin{gathered}
w_{r}(t)=P_{t} w_{r}=\sum_{n=1}^{N} \int_{-\infty}^{t} g_{n, r}(u) d z_{n}(u), \\
G_{r}(t)=E\left|w_{r}(t)\right|^{2}=\sum_{n=1}^{N} \int_{-\infty}^{t} g_{n, r}(u) d F_{n}(u) .
\end{gathered}
$$

We shall now show that the sequence $w_{1}, \ldots, w_{N}$ satisfies the conditions of the Lemma.

In order to show that the cyclic subspaces $C\left(w_{1}\right), \ldots, C\left(w_{N}\right)$ are orthogonal, it will be sufficient to show that $w_{r}(t) \perp w_{s}(u)$ for $r \neq s$ and all $t, u$. Clearly we may suppose $t \leqq u$, and then have by (6)

$$
E w_{r}(t) \overline{w_{s}(u)}=\sum_{n=1}^{N} \int_{-\infty}^{t} g_{n, r}(v) g_{n, s}(v) d F_{n}(v)=0 .
$$

Further we evidently have, since all the $w_{r}$ are elements of $H(\mathbf{x})$,

$$
H(\mathbf{x}) \supset C\left(w_{1}\right) \oplus \ldots \oplus C\left(w_{N}\right) .
$$

In order to show that the two members of this relation are identical, we have to show that every element of $H(\mathbf{x})$ belongs to the vector sum of the $C\left(w_{r}\right)$. Now by hypothesis every element of $H(x)$ is of the form

$$
y=\sum_{n=1}^{N} \int_{-\infty}^{\infty} c_{n}(t) d z_{n}(t)
$$

where $c_{n}(t)$ belongs to $L_{2} F_{n}$. By (6) we have for every $n$ and $t$

$$
\sum_{r=1}^{N} g_{n, r}(t)=1 \quad \text { or } \quad 0 .
$$

In the set of values of $t$ where this sum is equal to zero, it follows from the definition of $g_{n, r}(t)$ that $F_{n}^{\prime}(t)=0$, so that the integral of $c_{n}(t) d z_{n}(t)$ over this set is zero. Thus we have

$$
y=\sum_{r=1}^{N} \sum_{n=1}^{N} \int_{-\infty}^{\infty} c_{n}(t) g_{n, r}(t) d z_{n}(t)
$$

Now define a function $b_{r}(t)$ by writing

$$
b_{r}(t)=\left\{\begin{array}{ccc}
c_{n}(t) & \text { when } & g_{n, r}(t)=1 \\
0 & \text { when } & g_{n, r}(t)=0
\end{array}\right.
$$

By $(7)$ this $b_{r}(t)$ is uniquely defined a.e., and we have

$$
y=\sum_{r=1}^{N} \int_{-\infty}^{\infty} b_{r}(t) \sum_{n=1}^{N} g_{n, r}(t) d z_{n}(t)=\sum_{r=1}^{N} \int_{-\infty}^{\infty} b_{r}(t) d w_{r}(t)
$$

As it is easily seen that $b_{r}(t)$ belongs to $L_{2} G_{r}$, this shows that $y$ is an element of the vector sum of the $C\left(w_{r}\right)$, so that (5) is satisfied. 
It remains to show that (4) is also satisfied. We consider the variance functions $G_{r}(t)$ of the $w_{r}$ and have to show that $G_{r}>G_{r+1}$ for $r=1, \ldots, N-1$. Let $S$ be a set of positive measure such that

$$
\int_{S} d G_{r}(t)=\sum_{n=1}^{N} \int_{S} g_{n, r}(t) d F_{n}(t)=0 .
$$

It will then be proved that

$$
\int_{S} d G_{r+1}(t)=\sum_{n=1}^{N} \int_{S} g_{n, r+1}(t) d F_{n}(t)=0
$$

In every term of the sum in the last member, the integral extended over that part of $S$ where $g_{n, r+1}(t)=0$ is obviously zero. If $g_{n, r+1}(t)=1$, there is by (8) exactly one $m<n$ such that $g_{m, r}(t)=1$. But it follows from (9) that, for every $m$, the integral

$$
\int d F_{m}=\int F_{m}^{\prime} d \mathbf{F}
$$

extended over that part of $S$ where $g_{m, r}(t)=1$, and thus $F_{m}^{\prime}(t)>0$, is equal to zero. Consequently this set is a null set for the $\mathbf{F}$ measure, and the integral

$$
\int d F_{n}=\int F_{n}^{\prime} d \mathbf{F}
$$

extended over this set, is also equal to zero. As this holds for all $n$ and for all $m<n$, the relation (10) follows, and the proof of the Lemma is completed.

As already remarked above, the truth of the Theorem immediately follows from the two Lemmas.

\section{References}

1. Cramér, H., On the structure of purely nondeterministic stochastic processes. Arkiv för Matematik 4 (1961), 249-266.

2. Cramér, H., Stochastic processes as curves in Hilbert space. Teorija Verojatnostei $i$ eio Primenenija, 9 (1964), 193-204.

3. Cramér, H., Structural and statistical problems for a class of stochastic processes. S. S. Wlksi Lecture, Princeton University Press 1971.

4. KallianPuR, G. and Mandrekar, V., Multiplicity and representation theory of purely nondeterministic stochastic processes. Teorija Verojatnostei $i$ eio Primenenija, 10 (1965), 208-236.

Harald Cramér Sjötullsbacken 15 11525 Stockholm Sweden 